\title{
Use of Clustering to Improve the Layout of Gene Network for Visualization
}

\author{
Nasimul Noman \\ Dept. of Frontier \\ Informatics \\ The University of Tokyo \\ Japan \\ noman@iba.k.u-tokyo.ac.jp
}

\author{
Kouichi Okada \\ Dept. of Electronics \\ Engineering \\ The University of Tokyo \\ Japan \\ okada@iba.k.u-tokyo.ac.jp
}

\author{
Naoki Hosoyama \\ Dept. of Electronics \\ Engineering \\ The University of Tokyo \\ Japan \\ hosoyama@iba.k.u-tokyo.ac.jp
}

\author{
Hitoshi Iba \\ Dept. of Frontier \\ Informatics \\ The University of Tokyo \\ Japan \\ iba@iba.k.u-tokyo.ac.jp
}

\begin{abstract}
A very effective means to study the gene networks is visualization. With rapid increase of the size of gene networks, it has become more realistic to identify the collaborating genes in the network, which will facilitate the behavioral study of the groups and the network as a whole. In our previous paper, we presented a layered approach for visualizing gene regulatory networks. In this paper, we present a 3D layout model for visualizing gene networks, which clusters the correlated genes depending on their causal relationships. To demonstrate the effectiveness of the approach, we visualize real gene networks of different sizes. The experimental results show the superiority and usefulness of the new model when compared with previous results.
\end{abstract}

\section{INTRODUCTION}

In recent years, the revolutionary advancement in molecular biology, particularly in high-throughput genomics and proteomics, continue to produce massive biological data. And this data opens the door of extensive research in the many domains of bioinformatics. Inference of network model from gene expression data that can estimate the behavior of the biological system has found a new dimension and the constructed network size is growing rapidly. The most effective way to study such a bulk of relational information is to visualize it. For this reason, a well-suited technique for effective visualization of such a large network is desirable.

The usefulness of a graphical representation of some information depends on its presentation. The information contained in a gene network can only be studied well when its layout is well-structured enough to grasp the inter-gene relation easily. But generating a viable layout of a large network manually is almost impossible. That's why the automated layout generation is desirable.

Identifying the natural clustering of nodes in a graph and treating them as a supernode or meta-node for a higher level graph or an abstract graph is a technique used for the reduction of visual complexity of a graph with large number of nodes [1]. For a biological network, clustering can clarify the mutual relationship among the interacting components thus improves the quality of represented information in terms of analytical capability. In our previous work, we had developed a 3 dimensional model for visualizing gene network. In this work, we have used clustering to improve the quality of the layout generated by our method. This new model and our enhanced visualization software have many distinguishing features most suitable for elegant visualization like:

1) In biological networks, a single gene is affected by many other genes and this gene in turn affects many other genes. Often genes influence each other in a localized manner. Our model is capable to identify such a faction of genes. This facilitates the study of the genes behavior because it is required to study the genes in a collective manner rather than isolate [2].

2) The genes are positioned carefully to avoid overlapping of genes and also the crossings of arcs were also minimized to enhance the visualization capability.

3) Since the network is arranged in a three dimensional space, it can be examined from a variety of directions and can also be viewed with arbitrary details by zooming in and out.

4) In order to make the relation between genes more easy to understand, we extended our previous 3-layer model into 5-layer.

5) For comprehensive study, the complexity of the network can be varied by displaying genes with a maximum number of relations.

6) A single gene in the whole network with its relationship with other genes is observable hiding the rest of the network. This is more effective for a network with large number of genes.

In our previous papers, we defined fitness functions in terms of the clarity and optimize the functions by means of stochastic search methods. In this paper, we have improved the fitness function by simplifying it for less complexity and adapting it for clustering model. In order to judge the effectiveness of clustering, we compare the performance of this model with previous model in terms of visual enhancement and the results are presented with discussion.

This paper is organized as follows. The next section gives background and fundamental facts about the work. After that, Section III explains our proposed technique. Section IV presents experimental results with the visualization of two real networks. Finally, the last section presents discussion and gives 
direction of future researches.

\section{Visualizing Gene Networks}

\section{A. Gene Network Models}

There have been several models proposed for gene regulatory networks, e.g., Boolean network [3], S-system [4], and Bayesian network [5]. Each of these models has its own characteristic approach to generate the network and different capability in terms of handling information. In most of these models, the relationship between genes is represented by an arc. And depending on the qualitative or quantitative information associated with each arcs of their graphs, they can be broadly classified into following three classes [6]:

1) Correlation Graph: This graph only contains the information about the positive / negative correlation between genes. Two related genes are connected with an undirected arc.

2) Cause Effect Graph: This graph represents the causality relationships among the genes. Causality is represented by a directed arc, whose direction shows the cause- effect relation between genes.

3) Weighted Graph: Some qualitative meaning is attached to each arc of the graph, e.g., S-system or Bayesian networks.

In this paper, we have used cause-effect graph to develop and visualize our model, considering the nature of estimated data on the genetic network, which can be estimated at present.

\section{B. Layout Policy}

The positioning of nodes may have perceivable effect on the visibility and understandability of the network. Any haphazard positioning of a graph is very easy but it becomes least useful in terms of lucidity. A simple example of a small graph of 6 nodes and 8 arcs explains it significantly (Fig. 1). Just because of the difference in positioning of nodes the figure on the right is much easy to interpret.

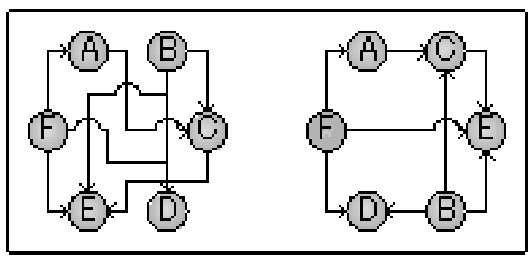

Fig. 1. Easy sample of useful layout

The situation goes beyond capability when the number of nodes and arcs increase exceedingly. This is why careful positioning of nodes is important. In order to improve the expressiveness of graph, some guidelines should be followed at the time of the laying out. The three principles followed by our model to improve the cogency of the layout are summarized in Table I with illustrative figures. The first column contains the regulation to be followed, the second column contains a poor layout violating the regulation and the third column presents a good layout that satisfies the regulation.
TABLE I

USEFUL NODE LAYOUT TECHNIQUES

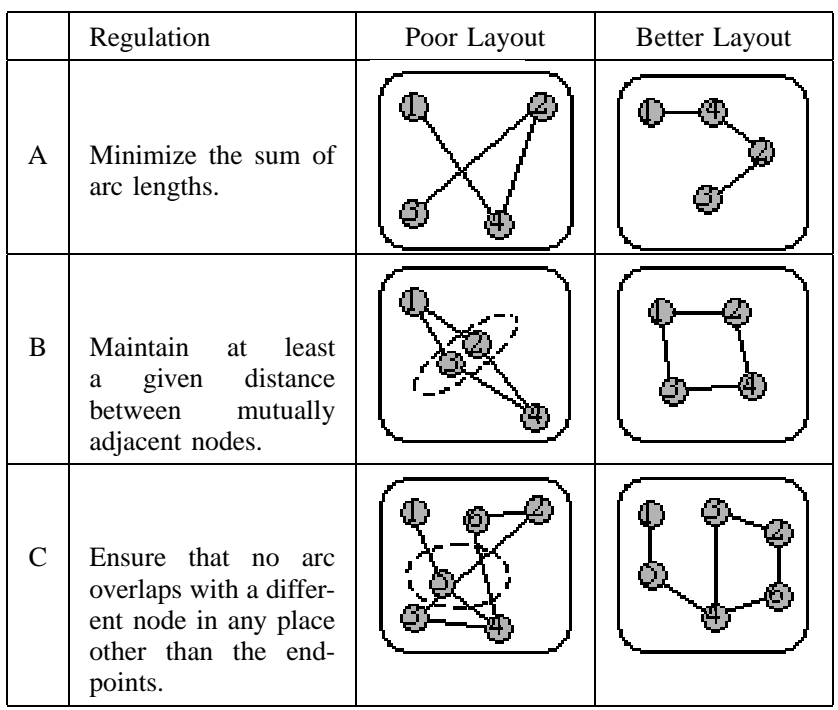

\section{Proposed Layout Method}

\section{A. Basic Idea}

The regulatory relationships among the genes are represented in a gene network. Since these interactions among the genes are often in collective manner, we use a clustering method to assemble the congregating genes in some clusters. Then genes in each cluster are partitioned into three layers (named green, red and blue) depending on their relationship with other genes. Then genes of each layer are arranged in a hexagonal lattice format. After initial layout we determine the score of the whole network according to the defined score function and use some stochastic search method to improve the score and thereby the arrangement of the network. The basic technique is illustrated in Fig. 2.

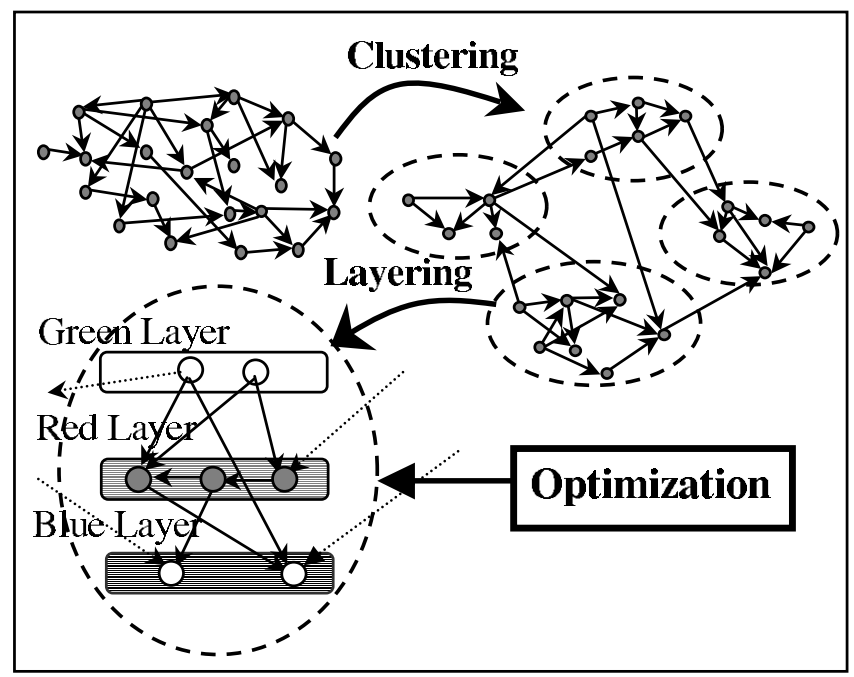

Fig. 2. The basic steps of layout 


\section{B. Clustering Method}

For graph clustering problem, many sophisticated algorithms have been developed which are applicable for different applications. The two most popular schemes are: k-path clustering algorithm and MCL algorithm [7]. But since we want to have a control on the number of clusters in which we partition the gene network, we used the multi-way ratio cut algorithm with stochastic search for clustering purpose [1].

First, we determine the number of clusters using the following scheme. Suppose the number of genes in the network is $n$. we calculate the value $r^{\prime}=\lfloor\sqrt{n / 5}\rfloor$. This is the probable number of clusters. But for giving a symmetric arrangements of clusters in visualization space, we find the value $t=\left\lfloor\sqrt{r^{\prime}}\right\rfloor$ and calculate $(i) t^{2}$ and $(i i) t(t+1)$. Between $(i)$ and $(i i)$ whichever is closer to $r^{\prime}$ is the number of clusters $r$.

After determining the number of clusters, we use the multiway ratio cut algorithm to partition the gene network into $r$ clusters. The basic principle of this algorithm is to minimize the number of cut edges between the clusters. The fundamental algorithm exhaustively searches for the minimum number of cut edges among clusters [1]. But since the sizes of the gene networks are very large, we adapted the algorithm using stochastic searches for efficiency. Our stochastic search version of the multi-way ratio cut algorithm is briefly described here.

Let us assume, the gene network is represented by the graph $G=(V, E)$. The value $c_{i, j}$ will be 1 if there is relation between the gene $i$ and $j$ otherwise 0 . Now we want to partition the network into $r$ clusters $\Gamma=\left\{V_{1}, V_{2}, V_{r}\right\}$. The ratio value of these $r$ clusters is

$$
\begin{aligned}
r v & =R\left(V_{1}, V_{2}, \ldots ., V_{r}\right) \\
& =C\left(V_{1}, V_{2}, \ldots \ldots, V_{r}\right) /\left(\left|V_{1}\right| \times\left|V_{2}\right| \times \ldots . . \times\left|V_{r}\right|\right)
\end{aligned}
$$

where

$$
C\left(V_{1}, V_{2}, \ldots . ., V_{r}\right)=\frac{1}{2} \sum_{p=1}^{r} \sum_{i \in V_{p}} \sum_{j \notin V_{p}} C_{i j}
$$

We use equation (1) as the score value of the clusters $\Gamma$ and use hill climbing and simulated annealing search to optimize this score. The search algorithms used are as follows:

Step 1: Initially, place all genes randomly in different clusters, and calculate the score $r v$.

Step 2: Randomly select two clusters $V_{a}, V_{b}$ where $V_{a} \neq V_{b}$.

Step 3: Choose a gene $g$ in cluster $V_{a}$ randomly, and removing from $V_{a}$ put it in $V_{b}$.

Step 4: (a) For Hill Climbing: If the score $r v$ is decreased then leave $g$ in $V_{b}$ accepting the new score as $r v$ otherwise return $g$ in $V_{a}$ and retain the old score.

(b) For Simulated Annealing: If the score $r v$ is decreased or $\rho<e^{-\triangle r v / T}$ (where $\rho$ is a random number between 0 and 1 and $\triangle r v$ is the score difference, $T$ is simulated annealing parameter) then leave $g$ in $V_{b}$ accepting the new score as $r v$ otherwise return $g$ in $V_{a}$ and retain the old score. Update $T$ by $T=k T(0<k<1, k: S A$ parameter $)$.
2.

Step 5: If the search is converged then stop. Else go to Step

\section{Layering Scheme}

With the increase of number of genes in network, the positioning of all genes in 2D space for clear visualization has become almost impossible. Therefore we have proposed a multiple 2D layered model where the genes are positioned in multiple 2D layers, which are in turn positioned in 3dimensional space. Depending on their causal relationships genes are classified into three categories.

Parent Only: This category contains those genes which are not regulated by other genes but regulate some genes.

Parent \& Child: There are some genes which control other genes and are being regulated by some others.

Child Only: The genes, which don't regulate any other gene but only are regulated by other genes, belong to this category

We place all the genes belonging to parent only category in the same layer and call it the Green Layer. Similarly parent \& child and child only genes are placed in separate layers and their layers are named as Red Layer and Blue layers respectively. The layering of genes is explained in Fig. 3. Our layering scheme puts the green layer as the top layer, red in the middle and blue in the bottom in 3D space.

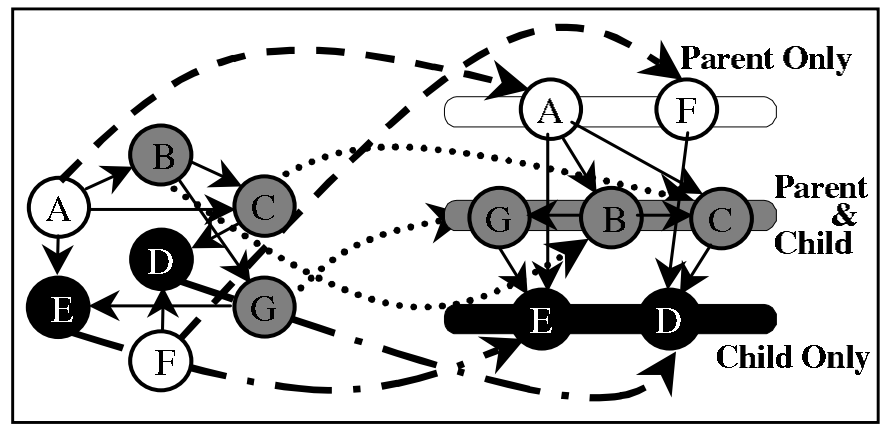

Fig. 3. Layering of genes

In order to make the red-to-red gene connections easier to understand in our extended model, we partition the middle (red) layer into three sub layers. The strategy of sub-layering is as follows: if a gene in red layer is connected with too many genes in green layer then it is to be brought closer to green layer i.e. it is to be placed in upper sub layer of red layer. Similarly if a gene in red layer is connected with too many blue genes then it is to be brought near to blue layer i.e. it is to be placed in the lower sub-layer of red layer. The rest of the red genes are kept in the middle sub-layer of the red layer. And with this 5 layer model visualization is enhanced incredibly.

\section{Positioning of Nodes in Layers}

In each layer of each cluster, the nodes are positioned on the edges of co-centric regular hexagon(s) with in a circle. Hexagon(s) are placed after equal interval with in the circle. Each hexagon has different radius where the outermost hexagon has radius equal to the circle (Fig. 4). The number 
of hexagons, on which the nodes to be positioned, is equal to the number of nodes on outermost hexagon segment, both of which depend on the number of nodes to be arranged in the layer of the cluster. Suppose that the number of nodes is $n$ and that the number of nodes in each segment is $m$. In order to find the most compact structure, we derive the minimum value of $m$ satisfying the following the equation (3). The larger the value of $m$, the more nodes are included in the layer.

$$
3(m-1) m+1<n \leq 3 m(m+1)+1
$$

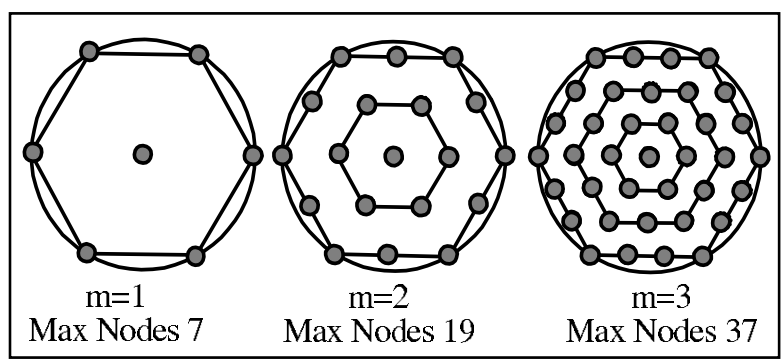

Fig. 4. Positioning of genes in different layers of clusters

Since there are many layers of genes in each cluster, the revelation of internal layers may be hindered by the positioning of genes in the outer layers. That's why the lattices of nodes in each cluster are rotated by an angle of 20 degree from the adjacent lattice layers. This is illustrated in Fig. 5. This rotation also offers the increase of the number of nodes that can be laid out in proportion to the number of layers.

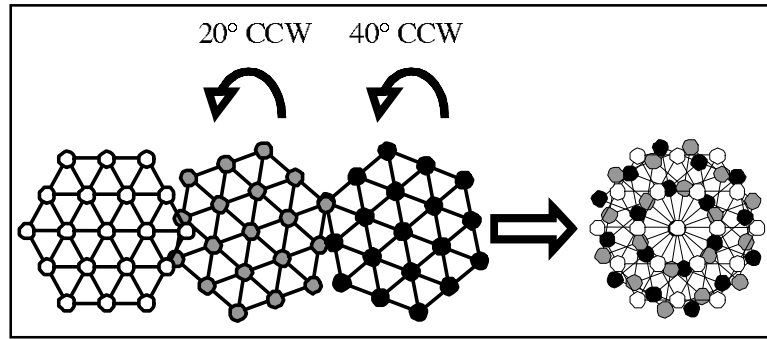

Fig. 5. Rotation of layers for easy visualization

\section{E. Evaluation Function}

The criteria for obtaining good layout of graphs for visualization were set in section II. The model described in the previous subsections meets the first two conditions directly and for our model the third condition is the logical consequence of the first two conditions up to some extent. So careful positioning of genes in layers of each cluster can avoid the crossing of arcs and genes and thus enhance the quality of positioning of the graph in terms of visualization. In this format, the layout problem for a graph can be considered as an optimization problem in which a scale to be defined that assess the quality of positioning of nodes and the scale to be optimized searching across the graph.

Since our model is a multiple 2D layered model in 3D space, we safely projected the $3 \mathrm{D}$ space in to $2 \mathrm{D}$ space for the sake of simplification. Then we define the "distance" between two genes as score of the relation between two genes. Therefore according to our strategy the smaller score value indicates the better the positioning. Here "distance" is not Euclidean distance. For reducing the computation burden we defined the "distance" between two genes (i.e the score of their relation) as

$$
S_{\text {node }}(a, b)=\frac{\left|a_{x}-b_{x}\right|+\left|a_{y}-b_{y}\right|}{\sqrt{\operatorname{deg}(a) \times \operatorname{deg}(b)}}
$$

Here $a$ and $b$ are the nodes connected to the ends of the arcs with coordinates $\left(a_{x}, a_{y}, a_{z}\right)$ and $\left(b_{x}, b_{y}, b_{z}\right)$ respectively. And $\operatorname{deg}(a)$ and $\operatorname{deg}(b)$ represents the degree of node $a$ and $b$ respectively. This score function is different from our previous scoring functions [6], [8] and adapted to the clustering scheme. This is explained using the Fig. 6. Here two arrangements are showed for a set of 5 nodes $A, B, C, D, O$, where the number beside each node represents its degree and number beside each arc represents its score value. Definitely Case 2 is better than Case 1 for clustering if node $B$ belongs to different cluster than the other 4 nodes. Our new score function distinguishes between these two arrangements and scores Case 2 lower than Case 1.

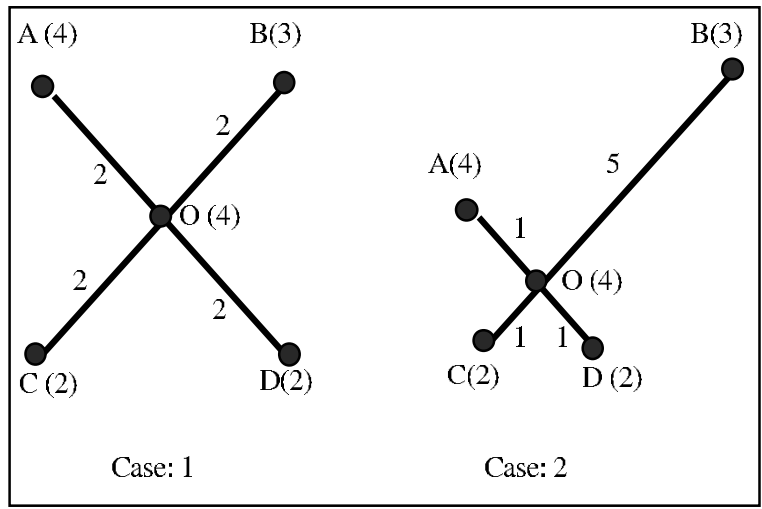

Fig. 6. Improved score functioin adapted to clustering

Basically, this is a problem which requires minimizing the distance. The score of the entire network is expressed by equation (5) which is the sum of each arc score [12].

$$
S=\sum S_{\text {node }}=\frac{1}{2} \sum_{\forall a} \sum_{\forall \text { linked to } a} S_{\text {node }}(a, b)
$$

A layout that minimizes this value is desirable. Since exhaustive search is unrealistic for a huge network having hundreds nodes we used stochastic searches to optimize the score.

\section{F. Stochastic Hill-Climbing Search}

Since the cluster and layer of each node is determined beforehand and is permanent, it is conceivable to swap two nodes' positions in the same cluster with the target to improve the fitness score of network gradually. This is the strategy of hill-climbing search. More precisely, we follow the process described below: 
Step 1. Initially, position all the nodes of each layer of each cluster randomly in the predetermined coordinates.

Step 2. Randomly select a cluster $r$.

Step 3. Choose a lattice point $p$ in cluster $r$ at random. This $p$ may or may not contain a gene.

Step 4. Choose randomly another lattice point $q$ in the cluster $r$ with $p$. We assume that at least one of these $p$ and $q$ contains a gene.

Step 5. Derive the new score if $p$ and $q$ are swapped. If the score is decreased, then swap $p$ and $q$.

Step 6. If the search is converged, then stop. Else, go back to Step 2.

We calculate the equation (5) in order to derive the score value in Step 5. During calculation of score difference we take the advantage of the fact that only two nodes are swapped and the rest of the network remains the same, i.e., the score for the rest also remains the same.

\section{G. Simulated-Annealing Search}

The simple hill-climbing method often falls into local optima. Therefore, we use a more robust stochastic search method, i.e., simulated annealing search. The algorithm is similar to hill climbing algorithm with some modification in step 5. For this search method, Step 5 is redefined as follows:

Step 5. a. Derive the new score if $p$ and $q$ are swapped. Generate a random number $\rho$ between 0 and 1 . If $\rho \leq e^{-\triangle S / T}$ or $\triangle S<0$ holds for the score difference $\triangle S$, then swap $p$ and $q$.

b. The temperature parameter is updated according to the following equation: $T \leftarrow k T(0<k<1)$.

Note that in the original Step 5 of hill climbing search, a simple direction to decrease the length is chosen. By using simulated annealing, we swap nodes $p$ and $q$ if the score value decreases with this swapping. In addition, even if the score value increases, the swapping is carried out with a certain probability. The probability is modified so that the higher the temperature $T$, the more often the swapping is accepted. The temperature is decreasing in a geometric progression. At the end, the search becomes equivalent to simple hill-climbing.

\section{EXPERIMENT \& RESULT}

\section{A. Data Sets}

In order to justify the effectiveness of the proposed model, we applied our method into two gene networks of different sizes. We used (5) as score function for evaluating the performance of the two algorithms mentioned above. The extents of the used gene networks are presented in Table II. Data sets $A$ and $B$ are real data inferred by a Boolean network.

\section{B. Results of Experiments}

In our experiment, there are two stages (1) Clustering and (2) Layout where the result of the first stage is used as the input of the second stage. In each stage, we used two stochastic search algorithms namely Hill Climbing (HC) and Simulated
TABLE II

DATA SETS

\begin{tabular}{|l|lll|}
\hline & \#of genes & \#of relations & Source \\
\hline A & 82 & 84 & {$[9]$} \\
\hline B & 552 & 2953 & {$[10],[11]$} \\
\hline
\end{tabular}

Annealing (SA) to find optimal score. We tried all possible combinations of these algorithms in these two stages for both network and their results are presented in the Table III and Table IV. Here the data in the Table III and Table IV is the average values of evaluation functions over 20 runs.

TABLE III

RESULTS FOR 82 GENE NETWORK

\begin{tabular}{|llll|}
\hline $\begin{array}{l}\text { Clustering } \\
\text { Algorithm }\end{array}$ & $\begin{array}{l}\text { Laying out } \\
\text { Algorithm }\end{array}$ & Score & Time \\
\hline HC & HC & 14.7343 & $00: 00: 07$ \\
\hline HC & SA & 12.9931 & $00: 00: 13$ \\
\hline SA & HC & 12.7962 & $00: 00: 07$ \\
\hline SA & SA & 11.0918 & $00: 00: 13$ \\
\hline
\end{tabular}

TABLE IV

RESULTS FOR 552 GENE NETWORK

\begin{tabular}{|llll|}
\hline $\begin{array}{l}\text { Clustering } \\
\text { Algorithm }\end{array}$ & $\begin{array}{l}\text { Laying out } \\
\text { Algorithm }\end{array}$ & Score & Time \\
\hline HC & HC & 784.6143 & $00: 02: 58$ \\
\hline HC & SA & 808.2950 & $00: 04: 15$ \\
\hline SA & HC & 950.9012 & $00: 03: 08$ \\
\hline SA & SA & 955.6906 & $00: 04: 25$ \\
\hline
\end{tabular}

From the above tables, it is found that result may vary significantly for different combinations of the algorithms. According to results, it takes only a few seconds to converge for a small network like data set $A$. On the other hand, for the larger sets such as $B$ it takes much longer time to settle down. In other words, the convergence time is exponentially related with the number of nodes and arcs of the network, which shows the significance of using efficient search algorithm to solve this computational burden.

One important observation in the experiment is the effect of clustering in the final score of the network. In general, it was found that the final score of the network obtained from layout stage depends significantly on the clustering score obtained from the first stage of clustering. This is presented in Fig. 7.

From Fig. 7, it is clear that if we can generate a good clusters of the gene network then we will find a good score value from the layout stage i.e. we find a positioning of nodes that is good for visualization. This is also shown in next subsection using snapshots of networks. 


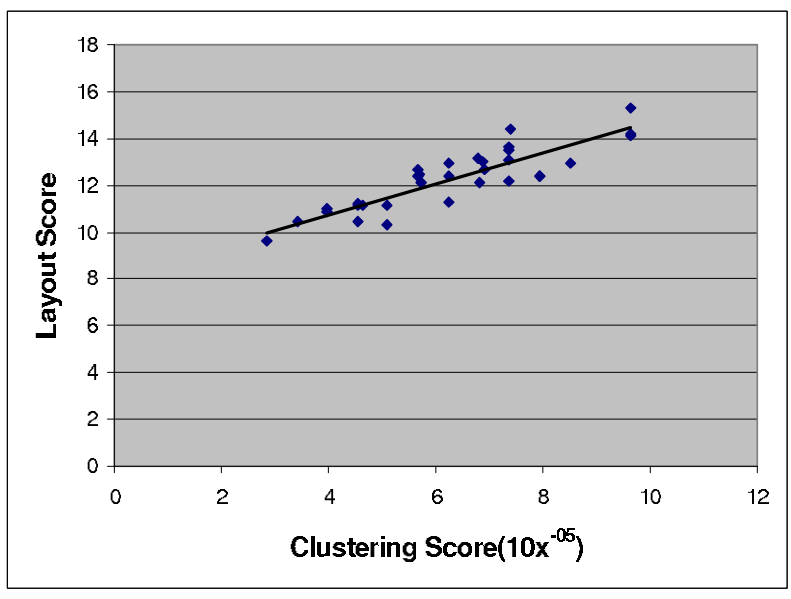

Fig. 7. Effect of clustering on layout score

\section{Visualization Results}

Most effective way to assess the quality of the layout of a gene network is to visualize it. For this reason, we developed a visualization software implemented in JAVA 3D platform and used it to compare the layout generated by clustering method with that of our previous model (without clustering). We use both of our data sets for this purpose and the screen shots from the software are presented here for comparison.

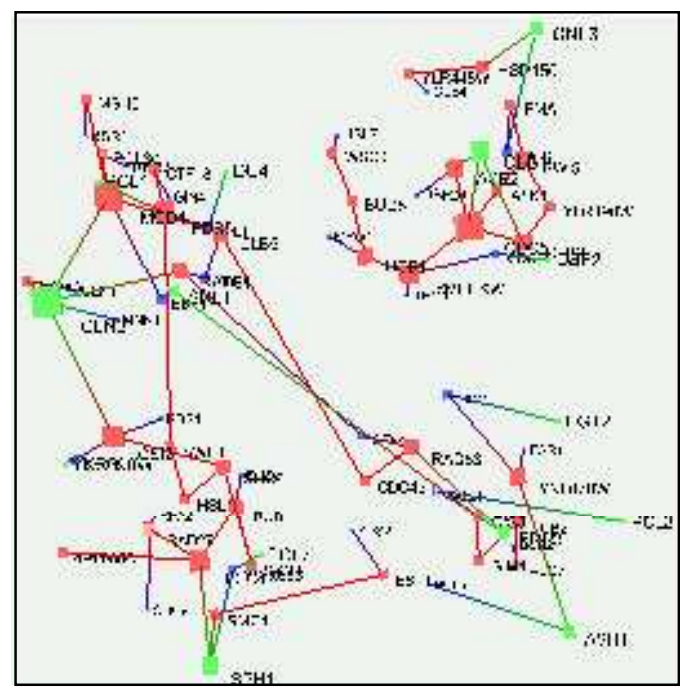

Fig. 8. Layout of 82 gene network using 4 clusters

Fig. 8 and Fig. 9 compares the layout generated by clustered model (Fig. 8) with non-clustered model (Fig. 9) of 82 gene network. Each gene is represented by a cube whose volume is proportional to its degree, i.e. the more nodes a node are connected to, the larger the cube used to present it.

It is evident from the figure that the study of the network is much easy with the new layout (Fig. 8). The clustered model positioned the nodes in such a way that the relationships among the genes are now easy to capture, more over the number of crossing of arcs and nodes is minimized. Further more it was successful to extract a clique from the whole network which facilitates the observation of this group of genes independently.

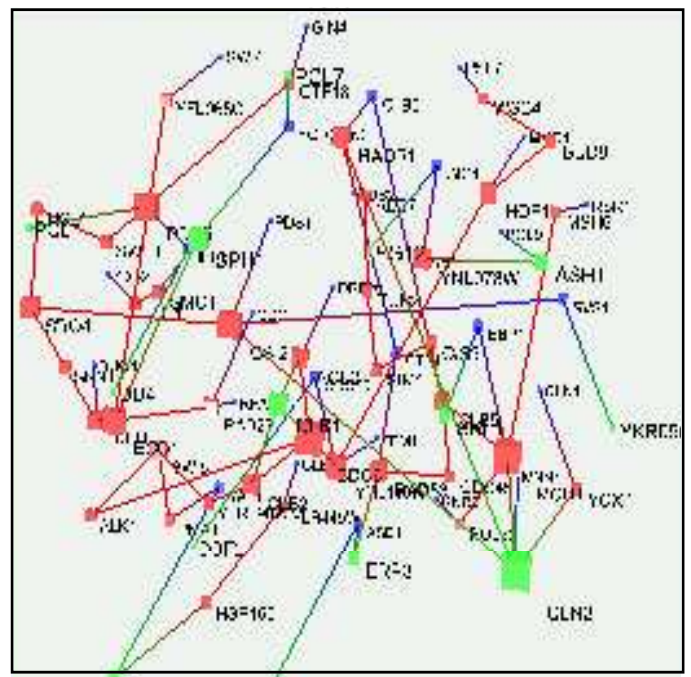

Fig. 9. Layout of 82 gene network without clustering

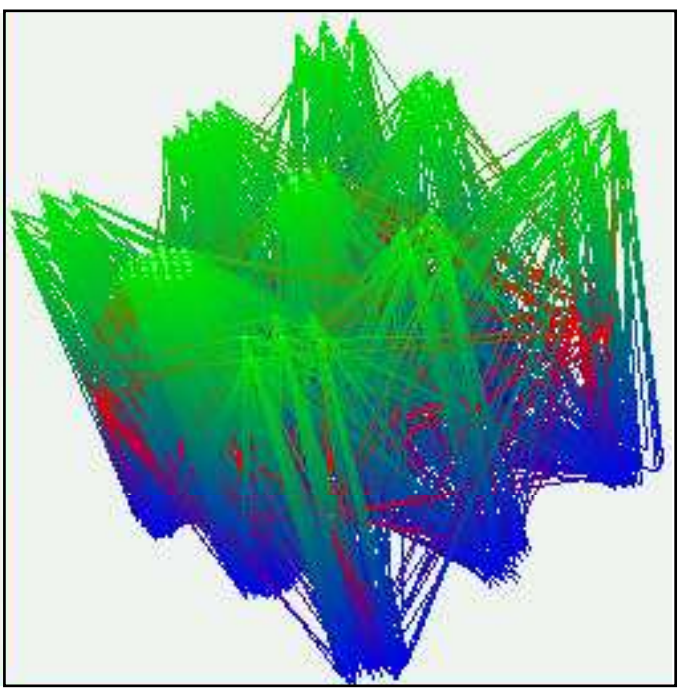

Fig. 10. Layout of 552 gene network using 9 clusters

The 552 gene network is compared similarly in Fig. 10 and Fig. 11. For capturing the whole network we zoomed out therefore the relationships among the genes are not clear because of too many nodes and genes. But from Fig. 10 and Fig. 11 it is clear that the clustered model is much suitable for studying the genes in the network.

From Fig. 10 and Fig. 11, it is obvious that it is difficult to grasp the whole structure for such a large network if all the nodes and arcs are drawn. To deal with this problem, our software provides the facility of observing a single gene with its neighboring genes and their relations. This is shown in Fig. 12 and it is clear from the figure that all the genes that are closely connected are put in the same cluster which exists from top to bottom direction. 


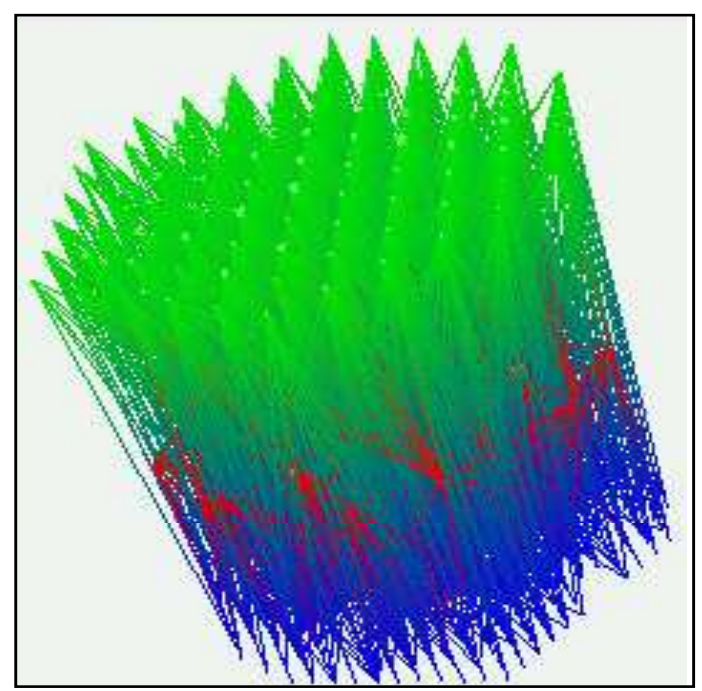

Fig. 11. Layout of 552 gene network without clustering

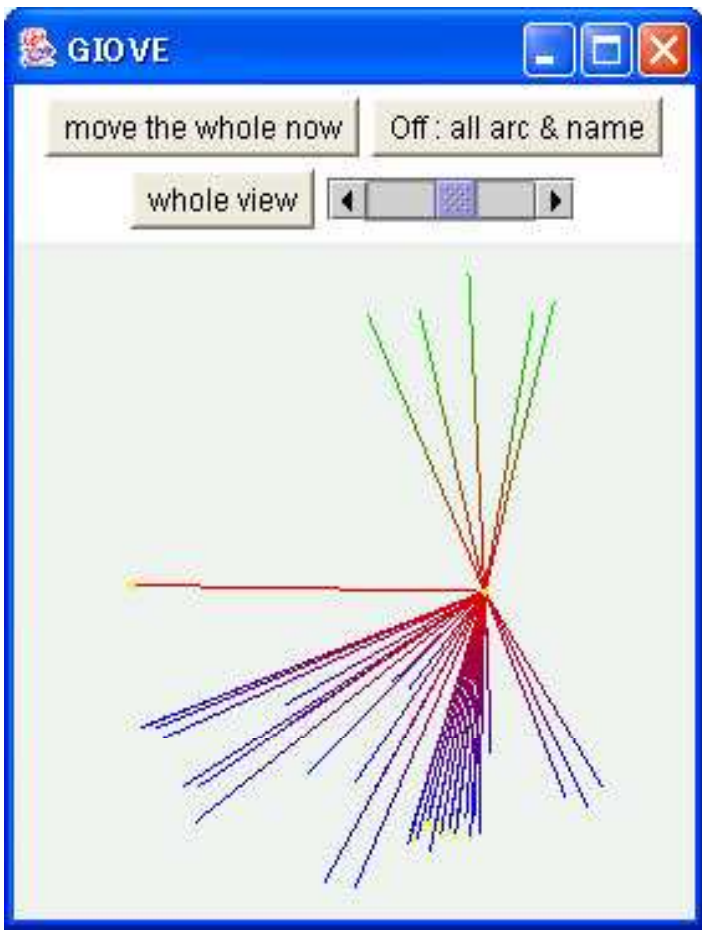

Fig. 12. A focused gene with its neighbors

As mentioned in previous subsection, the quality of clusters has a substantial effect on the quality of layout generated. This fact is also presented here in terms of visualization in Fig. 13. As the figure reveals if the quality of clustering is not good then genes are scatteredly positioned and the number of crossings among nodes and arcs are very high and the whole network remains obscure. And as the quality improves (from Scenario 1 to Scenario 4 in Fig 13) the closely acting genes are brought nearer with in the same cluster and the whole image looks more organized, ordered and clear, which is easy to understand.

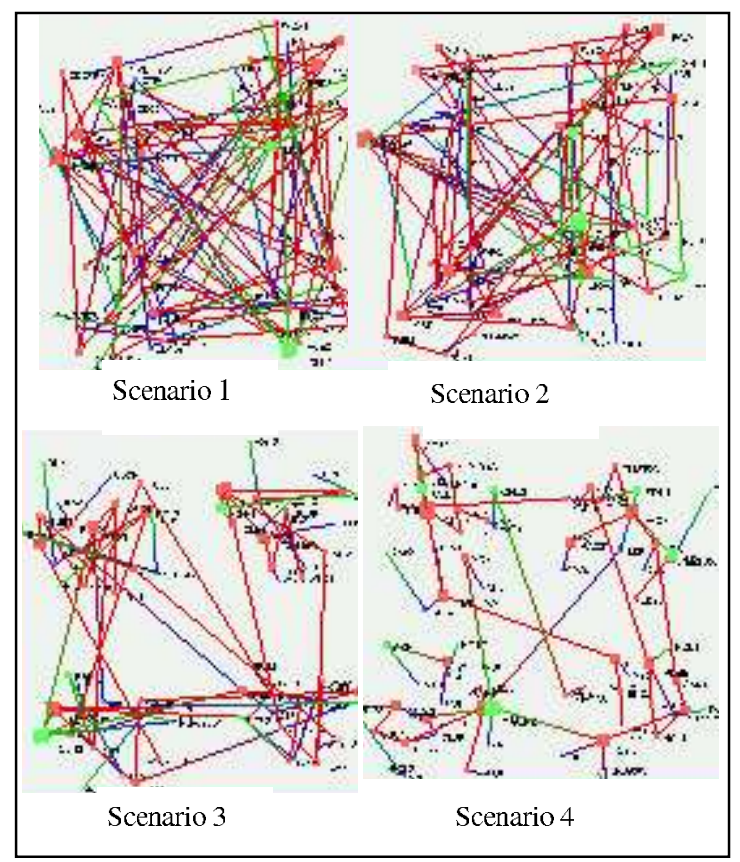

Fig. 13. Good clustering generates good layout

\section{User Interface or Functions}

A user can manipulate the visualization process with a mouse or touch pad. For instance, he or she can look over the network from a variety of directions. In addition, the following features are provided for enhanced functionality and user-friendly interface:

- New functions

- Any gene together with its relations with adjacent genes in the network can be focused by the user (Fig. 14a).

- Network can be displayed with all genes and relations or with the genes only (no arc).

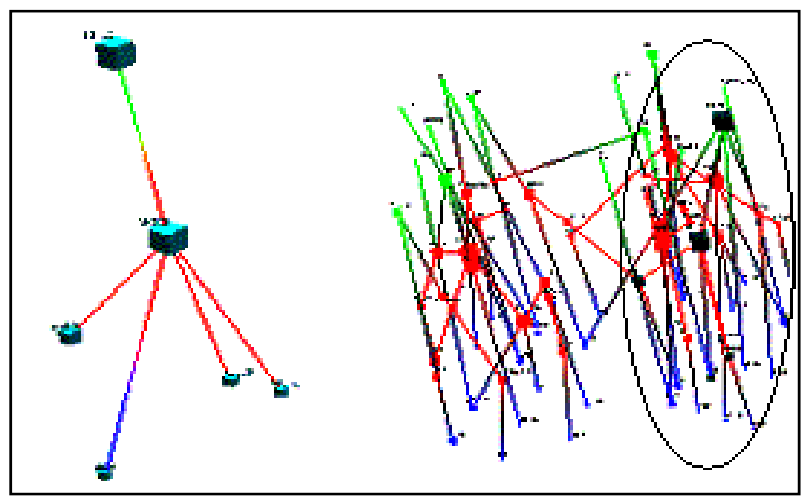

Fig $14 \mathrm{a}$

Fig 14b

Fig. 14. Focused gene with its neighbors is easy to observe

\section{- Improved functions}

- Using the operating scrollbar the complexity of the network can be controlled by showing the genes 
(with their names) with a minimum number of relations only and eliminating the rest of the network. Using this function user can easily identify the hub genes.

- A focused gene with its relation to the adjacent genes (with their names) can be displayed both eliminating the rest of the network (Fig. 14a) and in presence of the rest of the network (Fig. 14b).

- Any gene can be repositioned according to user's preference.

- 5-layer presentation of the whole gene network is easy to understand than 3-layer presentation, especially for red genes(Fig. 15).

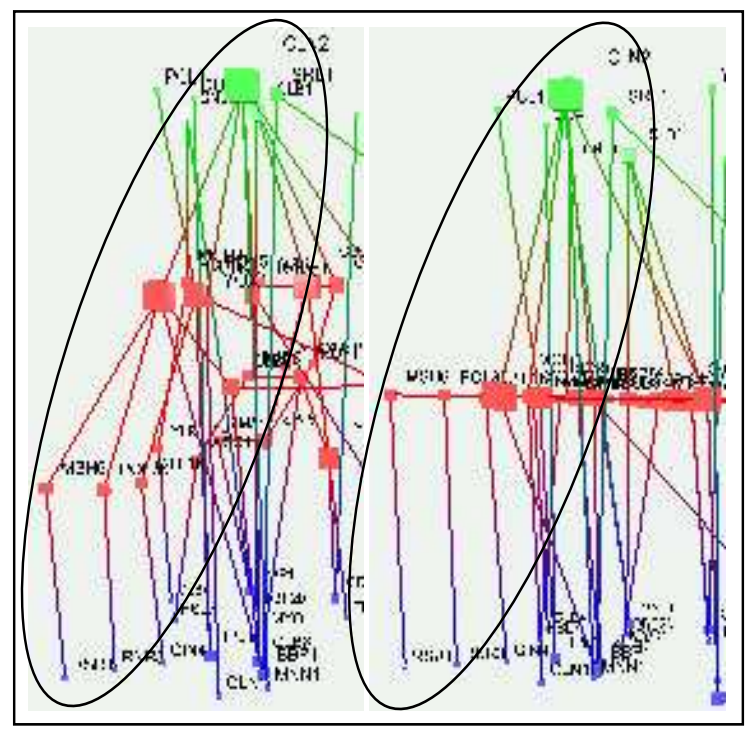

Fig. 15. Comparing 5-layer model with 3-layer model

The software is available from the following URL: http://www.iba.k.u-tokyo.ac.jp/ /okada/

\section{CONCLUSION AND FUTURE WORK}

For understanding the behavior of genes comprehensively, they should be studied in the cliques in which they act rather than individually. The most eminent way to partitioning the whole gene network into the collaborating groups is clustering. In this paper, we presented a layout model for gene regulatory networks that uses clustering to concentrate the genes into their working factions. The clustering was performed based on binary relation only. The proposed method, which is the clusterized version of our previous work, generates layout in $3 \mathrm{D}$ space which is most suitable for visualizing gene networks with hundreds of nodes. The proposed method was verified with two gene networks constructed from real data and the layout was found remarkably improved when compared with our previous method that does not use clustering. The new model generates the high-quality layout that is very suitable for the study of the gene behavior in the network. Although this model is specially suited for visualizing the gene regulatory networks, it may be useful to other applications where large networks need to be visualized.

We are working for enhancement of the model and software. Some qualitative or quantitative value of the relation as found in Bayesian network can be used to improve the layout of nodes. Methods for immediate detection and avoidance of crossings of nodes and arcs will improve the quality of layout. Use of GA and GP to search the optimal layout score can produce interesting results. Geometrical shapes other than hexagon such as sphere can be used for positioning the genes so that easier-to-view graph can be drawn by minimizing overlapping of nodes and arcs.

\section{REFERENCES}

[1] A. Sen and T. Roxborough, "Graph clustering using multi-way ratio-cut," Proceedings of Symposium on Graph Drawing (GD'97), pp. 291-296, Berlin, 1997.

[2] S. Ando, E. Sakamoto, and H. Iba, "Evolutionary modeling and inference of gene network," Information Sciences, vol. 145, no. 3-4, pp. 237-259, 2002.

[3] T. Akutsu et al, "Identification of genetic networks from a small number of gene expression patterns under the Boolean network model," The 4th Pacific Symposium on Biocomputing, pp. 17-28, 1999.

[4] H. Iba, and E. Sakamoto, "Inference of differential equation models by genetic programming," Proc. of the Genetic and Evolutionary Computation Conference (GECCO2002), pp. 788-795, 2002.

[5] Imoto et al, "Estimation of genetic networks and functional structures between genes by using Bayesian networks and nonparametric regression," The 7th Pacific Symposium on Biocomputing, pp. 175-186, 2002.

[6] N. Hosoyama, N. Noman, and H. Iba, "Layout search of a gene regulatory network for 3-D visualization," Proceedings of Genome Informatics, vol. 14, pp. 104-113, 2003

[7] S. van Dongen, "A new cluster algorithm for graphs," in 281, pages 42. Centrum voor Wiskunde en Informatica (CWI), ISSN 1386-3681, 311998.

[8] N. Hosoyama, and H. Iba, "3-D visualization of a gene retulatory network: stochastic search for layouts," Proc. of IEEE Conference on Electronic Commerce, pp. 1840-1847, 2003.

[9] T. Akutsu, S. Miyano, and S. Kuhara, "Algorithms for inferring qualitative models of biological networks," The 5th Pacific Symp on Biocomputing, pp. 290-301, 2000

[10] S. Aburanani et al, "Discovery of novel transcription control relationships with gene regulatory networks generated from multiple-disruption full genome expression libraries," DNA Research 10, pp. 1-8, 2003.

[11] C. J. Savoie et al, "Use of gene networks from full genome microarray libraries to identify functionally relevant drug-affected genes and gene regulation cascades," DNA Research 10, pp. 19-25, 2003.

[12] K. Okada, N. Noman, N. Hosoyama, and H. Iba, "3-D visualization software of a gene regulatory network," Proceedings of Genome Informatics (GIW2003), pp. 386-387, 2003. 